\title{
On scale separation in type II AdS flux vacua
}

\author{
Anamaría Font, ${ }^{a}$ Alvaro Herráez ${ }^{b}$ and Luis E. Ibáñez ${ }^{b}$ \\ ${ }^{a}$ Facultad de Ciencias, Universidad Central de Venezuela, \\ A.P. 20513, Caracas 1020-A, Venezuela \\ ${ }^{b}$ Departamento de Física Teórica and Instituto de Física Teórica UAM/CSIC, \\ Universidad Autónoma de Madrid, \\ Cantoblanco, 28049 Madrid, Spain \\ E-mail: afont@fisica.ciens.ucv.ve, alvaro.herraez@uam.es, \\ luis.ibannez@uam.es
}

Abstract: We study the separation of AdS and Kaluza-Klein (KK) scales in type II 4d AdS orientifold vacua. We first address this problem in toroidal/orbifold type IIA vacua with metric fluxes, corresponding to compactifications in twisted tori, both from the $4 \mathrm{~d}$ and $10 \mathrm{~d}$ points of view. We show how the naive application of the effective $4 \mathrm{~d}$ theory leads to results which violate the AdS distance conjecture, in a class of $\mathcal{N}=1$ supersymmetric models which have a 10d lifting to a compactification on $S^{3} \times S^{3}$. We show how using KK scales properly modified by the compact metric leads to no separation of scales with $M_{\mathrm{KK}}^{2}=\mathfrak{c}|\Lambda|$, with $\mathfrak{c}$ a numerical constant independent of fluxes. This applies with no need to keep non-leading fluxes fixed. We also consider a class of IIB models with non-geometric fluxes in which the effective field theory analysis seems to lead to a naive separation of scales and a violation of the AdS distance conjecture. It has a T-dual which again may be understood as a 10d type IIA theory compactified on $S^{3} \times S^{3}$. In this geometric dual one again observes that the strong AdS distance conjecture is obeyed with $M_{\mathrm{KK}}^{2}=\mathfrak{c}^{\prime}|\Lambda|$, if one takes into account the curvature in the internal space. These findings seem to suggest that all toroidal/orbifold models with fluxes in this class obey $M_{\mathrm{KK}}^{2}=\mathfrak{c}|\Lambda|$ with $\mathfrak{c}$ a flux-independent numerical constant.

KEYWORDs: Flux compactifications, Superstring Vacua

ArXiv EPRINT: 1912.03317 


\section{Contents}

1 Introduction 1

2 A class of type IIA vacua 2

2.1 Example 1, $M=0 \quad 5$

2.2 Example 2, $M \neq 0 \quad 7$

3 A class of type IIB vacua $\quad 8$

3.1 Example 3, $M=0 \quad 9$

4 Final comments $\quad 11$

\section{Introduction}

In the last few years there have been important efforts in trying to ascertain when a low energy effective field theory may be embedded into a consistent theory of quantum gravity. Failing to do so locates such a theory in the Swampland of theories. There is no general simple rule to learn when a theory is in the Swampland or not, see [1-3] for reviews. Frequently our knowledge of the Swampland territory is formulated in terms of conjectures which are then attempted to be tested in string theory, assuming that the latter is a consistent theory of quantum gravity. In the present paper we will be concerned with two such conjectures, both applying to AdS vacua. The first is the AdS/KK scale separation conjecture (ASSC) which states that in any AdS vacua there is no separation between the AdS and the lightest Kaluza-Klein (KK) scales, as stressed recently $[4,5]$ and earlier [6-8]. The second is the AdS distance conjecture (ADC) [5], which states that in AdS vacua with cosmological constant $\Lambda$, as $\Lambda \rightarrow 0$ there is an infinite tower of states with masses (in Planck units)

$$
m \simeq|\Lambda|^{\gamma},
$$

where $\gamma$ is a positive constant. Thus the limit $|\Lambda| \rightarrow 0$ is not smooth, but rather lies at infinite distance. A strong version of this conjecture states that $\gamma=1 / 2$ in the supersymmetric case. These two conjectures are not unrelated since for $\gamma=1 / 2$ they are actually equivalent (assuming that the tower predicted by the ADC is the KK tower, as in all the cases analysed in this paper). ${ }^{1}$

These conjectures have been tested in many string theory examples. However there is a class of type IIA $4 \mathrm{~d}$ orientifolds [11-16], which appear to violate both [5, 10, 17]. In particular there are certain examples with non-vanishing Romans mass which seem to yield

\footnotetext{
${ }^{1} \mathrm{~A}$ different $A d S /$ moduli scale separation conjecture posits that the lightest modulus cannot be heavier than the AdS scale $[9,10]$ and it is fulfilled in all our examples.
} 
theories in which the AdS and KK scales can be parametrically separated [12, 13]. It has been suggested that these models do not obey the strong ADC because the effective field theory fails to capture the backreaction effects of the background [5].

In this paper we revisit this issue and study specifically a class of $\mathcal{N}=1$ supersymmetric models which have an explicit uplift to a 10d theory compactified on $S^{3} \times S^{3}$ [18] (see also [19-21]). From the effective field theory point of view these are compactifications with metric fluxes. We find that using the effective action one obtains a violation of the strong ADC. However, when including information from the full $S^{3} \times S^{3}$ geometry one obtains that $M_{\mathrm{KK}}^{2}=\mathfrak{c}\left|V_{0}\right| / M_{\mathrm{P}}^{2}$, with $\mathfrak{c}$ a numerical constant independent from fluxes, and no scale separation. Given that $|\Lambda|=\left|V_{0}\right| / M_{\mathrm{P}}^{2}$, this is stronger than the original ADC in the sense that $\mathfrak{c}$ is flux independent and we are not making the non-leading fluxes small. We also study the case of a type IIB model with non-geometric fluxes which apparently displays AdS/KK scale separation. Although we do not have a geometric 10d interpretation of this model, it turns out to be mirror to a type IIA model of the above class, which admits a 10d uplift as a compactification on $S^{3} \times S^{3}$. In the dual frame one observes again that there is no scale separation and $M_{\mathrm{KK}}^{2}=\mathfrak{c}\left|V_{0}\right| / M_{\mathrm{P}}^{2}$, with $\mathfrak{c}$ flux independent. The results in these examples could hint that, after including backreaction effects, the class of supersymmetric AdS vacua in $[12,13]$ have all $M_{\mathrm{KK}}^{2}=\mathfrak{c}\left|V_{0}\right| / M_{\mathrm{P}}^{2}$, in agreement with the strong ADC.

Another interesting property that we have observed is that the ratio of the moduli masses to $|\Lambda|^{1 / 2}$ behaves as the ratio $M_{\mathrm{KK}} /|\Lambda|^{1 / 2}$. In fact, this also happens in nonsupersymmetric vacua found in the $4 \mathrm{~d}$ effective theory where our $10 \mathrm{~d}$ analysis does not directly apply. Nonetheless, in the non-supersymmetric case the $4 \mathrm{~d}$ results for the masses are consistent with no scale separation and the strong ADC.

The rest of this is structured as follows. In sections 2 and 3 we describe the IIA and IIB scenarios and discuss their main features. Section 4 is devoted to further observations.

\section{A class of type IIA vacua}

In this section we introduce the class of type IIA vacua in which the Swampland conjectures for different scales will be tested. They are $\mathcal{N}=1$ supersymmetric solutions of 10-dimensional type IIA supergravity with a warped product geometry

$$
d s_{10}^{2}=e^{2 A(y)} \hat{g}_{\mu \nu} d x^{\mu} d x^{\nu}+g_{m n} d y^{m} d y^{n},
$$

where $\hat{g}_{\mu \nu}$ and $g_{m n}$ are respectively the metrics of $\mathrm{AdS}_{4}$ and the internal space $\mathcal{M}_{6}$. On top there are background fluxes for the NS-NS 3-form $H$ and the RR $p$-forms, $F_{p}, p=0,2,4,6$. In particular, the Romans mass parameter corresponds to the flux of $F_{0}$. The complete form of such solutions was obtained in [22], extending work of [23, 24], and rederived in [25]. We now briefly summarize the results. The dilaton and the warp factor are constant, related by $\phi=3 A$. The internal space has $\mathrm{SU}(3)$ structure, characterized by a real 2 -form $J$ and a complex 3 -form $\Omega$, and only two non-vanishing torsion classes. In this paper we focus in the nearly Kähler case where only the class $\mathcal{W}_{1}$ is different from zero. The background fluxes are determined in terms of $J, \Omega, A$, plus constants denoted $m$ and $\tilde{m}$ in [25]. The 4-dimensional cosmological constant is determined to be $\Lambda=-3\left(m^{2}+\tilde{m}^{2}\right)$. The residual 
constraint from the Bianchi identity for $F_{2}$ can be fulfilled by adding smeared O6-planes and/or D6-branes. For certain choices of the parameters it is possible to avoid O6-planes, or even sources altogether [22-24].

In this paper we consider a particular example of nearly Kähler $\mathcal{M}_{6}$ given by $S^{3} \times S^{3}$. In $[18,21]$ it was shown that compactification on $\mathrm{AdS}_{4} \times S^{3} \times S^{3}$ admits an effective $4 \mathrm{~d}$ description by including appropriate geometric fluxes in the superpotential. Below we will first review the effective approach in $d=4$ and then discuss the lift to $d=10$ following [18].

The 4-dimensional setup is that of type IIA toroidal orientifolds. We will mostly follow the conventions of [13]. We consider a model with moduli consisting of three Kähler moduli $T_{i}$, together with complex structure moduli separated into the dilaton $S$ and three $U_{i}$. The potential for these fields is generated by RR, NS-NS and geometric fluxes. The fluxes are chosen so that there is a vacuum solution with $T_{i}=T$ and $U_{i}=U$. To simplify we can then restrict from the beginning to fields $S, U$ and $T$. According to the standard form of $\mathcal{N}=1$ supergravity, the scalar potential reads

$$
V=e^{K}\left\{K^{I \bar{J}} D_{I} W \overline{\left(D_{J} W\right)}-3|W|^{2}\right\},
$$

where $K^{I \bar{J}}$ is the inverse of $K_{I \bar{J}}=\partial_{I} \partial_{\bar{J}} K, D_{I} W=\partial_{I} W+K_{I} W$, and $I$ runs over $S, T, U$.

The Kähler potential is split as $K=K_{K}+K_{Q}$, where $K_{K}$ and $K_{Q}$ depend on Kähler and complex structure moduli respectively. In the large volume regime the Kähler piece is given by

$$
K_{K}=-\log (8 \mathcal{V})=-3 \log (T+\bar{T})-\log \mathcal{C} .
$$

In the second equality we have used that $\mathcal{V}$ is the volume of the internal manifold defined in terms of the Kähler 2-form through

$$
\mathcal{V}=\frac{1}{6} \int_{\mathcal{M}_{6}}^{J^{3}}=\frac{\mathcal{C}}{8}(T+\bar{T})^{3} .
$$

The normalization constant $\mathcal{C}$ will be specified by consistency with the 10-dimensional analysis. On the other hand, in the large complex structure limit

$$
K_{Q}=4 \phi_{4}=-\log (S+\bar{S})-3 \log (U+\bar{U})-2 \log \mathcal{C} .
$$

Here $\phi_{4}$ is the 4-dimensional dilaton related to the 10-dimensional one by $e^{\phi_{4}}=e^{\phi} / \sqrt{\mathcal{V}}$.

The flux-induced superpotential takes the form

$$
\frac{W}{\mathcal{C}}=e_{0}+3 i e T+3 c T^{2}+i M T^{3}+i h_{0} S-3 i h U-3 a S T-3 b T U .
$$

The parameters $M, c, e$ and $e_{0}$ correspond to fluxes of $F_{p}, p=0,2,4,6$, whereas $h_{0}$ and $h$ are fluxes of $H$. The terms mixing $T$ with $S$ and $U$ are due to the geometric fluxes denoted $a$ and $b$. Turning on geometric fluxes implies that the internal space is a so-called twisted torus having a basis of 1 -forms $\eta^{1}, \ldots, \eta^{6}$, satisfying relations such as $d \eta^{1}=-a \eta^{56}-b \eta^{23}$, with $\eta^{56}=\eta^{5} \wedge \eta^{6}$ and so on. The geometric fluxes $a$ and $b$ satisfy the Bianchi identity as checked by taking a further exterior derivative [13]. Consistency of the twisted torus structure requires quantized geometric fluxes [26]. The factor of $\mathcal{C}$ in $W$ arises from the 
normalization $\int_{\mathcal{M}_{6}} \eta^{1} \wedge \ldots \wedge \eta^{6}=\mathcal{C}$. As explained in detail in [13], the fluxes further contribute to tadpoles of the RR 7-form $C_{7}$ that couples to D6-branes and O6-planes.

It is convenient to work with dimensionless superpotential and Kähler potential. The units can be restored by inserting appropriate factors of the Planck mass $M_{\mathrm{P}}$, which actually appear writing the $4 \mathrm{~d}$ action in Einstein frame after dimensional reduction. In particular, in this way the scalar potential picks up a factor of $M_{\mathrm{P}}^{4}$. The relation between the string mass $M_{\mathrm{s}}$ and $M_{\mathrm{P}}$ reads

$$
M_{\mathrm{s}}^{2}=\frac{g_{s}^{2} M_{\mathrm{P}}^{2}}{4 \pi e^{2 A} \mathcal{V}}
$$

Here $g_{s}=e^{\phi}$ whereas $\mathcal{V}$ is the volume of the internal manifold. The warp factor enters because the metric that appears in the $4 \mathrm{~d}$ Einstein frame is $\hat{g}_{\mu \nu}$. In fact, $e^{2 A} \mathcal{V}$ corresponds to the warped internal volume for constant $A$. We will be interested in the Kaluza-Klein (KK) mass scale $M_{\mathrm{KK}}$. For constant warp factor it can be evaluated as

$$
M_{\mathrm{KK}} \sim \frac{M_{\mathrm{S}}}{e^{-A} \mathcal{V}^{1 / 6}} \sim \frac{g_{s} M_{\mathrm{P}}}{\mathcal{V}^{2 / 3}} .
$$

This estimate for $M_{\mathrm{KK}}$ corresponds to the lowest eigenvalue of the Laplacian in a well defined internal space $\mathcal{M}_{6}$. The largest size of $\mathcal{M}_{6}$ is approximated by $\mathcal{V}^{1 / 6}$, but it could be refined. In the above it is understood that $\mathcal{V}, A$, and $g_{s}$, are all evaluated at the moduli vevs.

AdS supersymmetric minima are straightforward to obtain by solving $D_{I} W=0$. The full results can be found in [13]. We will shortly analyze two particular examples with $M=0$ and $M \neq 0$. Our main purpose will be to study the various scales, namely the KK mass, the cosmological constant and the moduli masses. To this end we need to connect with the $10 \mathrm{~d}$ description of the vacua in order to determine the value of the constant $\mathcal{C}$ in the normalization of the internal volume.

In [18] it was shown that the AdS solutions found in the $4 \mathrm{~d}$ effective approach fully conform to the results predicted by the general $10 \mathrm{~d}$ analysis. To begin, the pair $(J, \Omega)$ of the twisted torus coincides with the nearly Kähler SU(3) structure of $S^{3} \times S^{3}$. In particular, the Kähler form can be written as

$$
J=\frac{t}{2 \sqrt{a b^{3}}} \sum_{i=1}^{3} \xi^{i} \wedge \hat{\xi}^{i},
$$

where $\xi^{i}$ and $\hat{\xi}^{i}$ are left-invariant forms of SU(2). Next, taking into account the moduli vevs found in $4 \mathrm{~d}$, the background fluxes can be expressed in terms of $(J, \Omega)$ precisely as dictated by the $10 \mathrm{~d}$ analysis. The parameters that determine the cosmological constant in the $10 \mathrm{~d}$ formulation are given by $m=\frac{M}{5} e^{4 A}$ and $\tilde{m}=\frac{3(c-M \operatorname{Im} T)}{t} e^{4 A}$, with $A=\frac{\phi}{3}$.

The explicit expression of the Kähler form $J$ allows to compute the volume of the internal manifold. It follows that $\mathcal{V}=\mathcal{C} t^{3}$, where

$$
\mathcal{C}=\frac{(4 \pi)^{4}}{\left(4 a b^{3}\right)^{3 / 2}}
$$

The dependence of $\mathcal{C}$ on the geometric fluxes wil be crucial in the ensuing discussion. We remark that this dependence can only be obtained from the full 10d solution, including 
the details of the backreacted internal geometry. Then, our philosophy in the following will be to compare the results obtained from the naive application of the purely $4 \mathrm{~d}$ EFT (i.e. without including the factors of $\mathcal{C}$ ) with those in which the full $10 \mathrm{~d}$ theory is used to determine the internal geometry as in [18], and the non-trivial dependence of $\mathcal{C}$ on the geometric fluxes is included.

\subsection{Example 1, $M=0$}

We first consider an example without Romans mass, namely without flux for $F_{0}$. To further simplify we set to zero the NS-NS fluxes $h_{0}$ and $h$, as well as the RR 4 -form flux $e$. In this case the Kähler axion and only one combination of the remaining axions are fixed as

$$
\operatorname{Im} T=0, \quad a \operatorname{Im} S+b \operatorname{Im} U=0 .
$$

The saxions $s=\operatorname{Re} S, u=\operatorname{Re} U$ and $t=\operatorname{Re} T$ are all stabilized at values

$$
s=\frac{2 c}{a} t, \quad u=\frac{6 c}{b} t, \quad t^{2}=\frac{e_{0}}{9 c}
$$

Without loss of generality we can choose $e_{0}>0$ so that necessarily $c, a, b>0$. Since the flux $e_{0}$ is not constrained by tadpole cancellation, it can be taken large to have large vevs for all saxions. On the other hand, to stay in perturbative regime with both couplings $e^{\phi_{4}}$ and $e^{\phi}$ small, it is necessary to take $c$ large. However, this coupling is constrained by cancellation of tadpoles that receive contributions proportional to $c a$ and $c b$ [13].

The cosmological constant is determined by the value of the potential at the minimum, denoted $V_{0}$. Inserting the above vevs for the moduli yields

$$
\frac{V_{0}}{M_{\mathrm{P}}^{4}}=-\frac{a b^{3}}{128 \mathcal{C} c^{2} t^{3}}=-\frac{27 a b^{3}}{128 \mathcal{C} c^{1 / 2} e_{0}^{3 / 2}}
$$

We remark that the cosmological constant calculated in the $10 \mathrm{~d}$ analysis matches the above result once the Planck units are restored [18].

The masses of the canonically normalized moduli are derived by diagonalizing the matrix $\frac{1}{2} K^{i j} \partial_{i} \partial_{j} V$ evaluated at the minimum. They are found to be proportional to $\left|V_{0}\right| / M_{\mathrm{P}}^{2}$. Specifically

$$
M_{\mathrm{mod}}^{2}=\left\{6, \frac{22}{27},-\frac{2}{3}, \frac{10}{3},-\frac{8}{27}, 0\right\} \frac{\left|V_{0}\right|}{M_{\mathrm{P}}^{2}} .
$$

The eigenvectors corresponding to the first (last) three entries are combinations of saxions (axions). As expected for a supersymmetric minimum, the negative eigenvalues are above the Breitenlohner-Freedman bound $m^{2} \geq-\frac{3}{4}\left|V_{0}\right| / M_{\mathrm{P}}^{2}[27]$. The zero eigenvalue correlates with the combination of axions that remains unfixed. The behavior $M_{\text {mod }}^{2} \sim\left|V_{0}\right| / M_{\mathrm{P}}^{2}$ is actually expected for generic tree-level flux vacua [10]. 
Let us now study the KK scale. Substituting the vevs in (2.8) gives

$$
\frac{M_{\mathrm{KK}}}{M_{\mathrm{P}}}=\frac{\left(a b^{3}\right)^{1 / 4}}{\mathcal{C}^{2 / 3} c^{1 / 4} e_{0}^{3 / 4}} .
$$

Here and below we will omit purely numerical factors to avoid cluttering. The relevant ratio to study both the ASSC and the strong ADC conjectures is $M_{\mathrm{P}} M_{\mathrm{KK}} /\left|V_{0}\right|^{1 / 2}$, which reads

$$
\frac{M_{\mathrm{P}} M_{\mathrm{KK}}}{\left|V_{0}\right|^{1 / 2}}=\frac{1}{\left(a b^{3}\right)^{1 / 4} \mathcal{C}^{1 / 6}} \text {. }
$$

It is then evident that the naive $4 \mathrm{~d}$ EFT approach could give misleading results. Without including the effect of $\mathcal{C}$, effectively setting $\mathcal{C}=1$ above, would lead to a flux dependent ratio between the $\mathrm{KK}$ and the cosmological constant scale. However, the value of $\mathcal{C}$ derived in the $10 \mathrm{~d}$ formulation, cf. (2.10), is such that the dependence of the ratio (2.16) on the geometric fluxes $a$ and $b$ drops out altogether. Thus, in the end we obtain the exact behavior predicted by the strong ADC not only in the limit $e_{0} \rightarrow \infty$, with other fluxes fixed, but for all values of the fluxes.

Let us observe that this first model serves to illustrate how the $10 \mathrm{~d}$ solution changes the purely $4 \mathrm{~d}$ picture in an interesting way, providing an exact flux cancellation in the relevant ratio of scales. However, we must remark, as noticed also in [10], that the $4 \mathrm{~d}$ EFT still did not have parametric scale separation nor gave a controllable counterexample to the strong ADC per se. The reason is that the two interesting limits in the fluxes in which this could occur, namely $a, b \rightarrow 0$ or $a, b \rightarrow \infty$ cannot be taken arbitrarily. The former cannot be explored because this family of solutions requires non-vanishing values of $a$ and $b$ and since they are quantized we cannot make them go continuously to zero. In the latter case, since the fluxes $a$ and $b$ enter the tadpole cancellation conditions, making them arbitrarily large would imply an unbounded number of D6-branes wrapping internal 3 -cycles, whose backreaction could bring the EFT out of control. In section 2.2 we will discuss an example in which there is no constraint from tadpole cancellation and the fluxes $a, b$ can take arbitrarily large values.

As seen from (2.14), in the $4 \mathrm{~d}$ EFT the moduli masses satisfy $M_{\bmod }=\mathfrak{n}\left|V_{0}\right|^{1 / 2} / M_{\mathrm{P}}$, with $\mathfrak{n}$ an order one (flux independent) constant. Such relation is actually valid without including the backreation of the geometric fluxes. We have further seen that the KK scale computed incorporating 10d effects has the same behavior as $M_{\text {mod }}$ with respect to the cosmological constant, in agreement with the strong ADC. As this happens in other examples of AdS vacua, it could be an indication, assuming that the strong ADC holds, that the ratio of the true KK scale to the cosmological constant can be estimated by the smallest ratio $M_{\text {mod }} /\left|V_{0}\right|^{1 / 2}$, even when the $10 \mathrm{~d}$ lift is not known in detail [10].

The example in this section, with equal configuration of fluxes, also admits classically stable non-supersymmetric vacua. The $4 \mathrm{~d}$ EFT results for the moduli and KK masses are qualitatively the same as in the supersymmetric vacuum. In Planck units $M_{\bmod } \sim\left|V_{0}\right|^{1 / 2}$ and the naive KK mass would match $\left|V_{0}\right|^{1 / 2}$ upon including the same volume correction as in the supersymmetric case. It would be interesting to see if there is a $10 \mathrm{~d}$ lift satisfying the general conditions spelled out in [28]. A 10d solution would presumably lead to a result for $M_{\mathrm{KK}}$ compatible with $M_{\text {mod }}$ up to numerical factors. 


\subsection{Example 2, $M \neq 0$}

We now turn to an example with $F_{0}$ flux and in which the geometric fluxes are not constrained by tadpole cancellation. To make our point it suffices to consider particular values for other fluxes. Concretely we take $e_{0}=0, e=0, h_{0}=3 c a / M$ and $h=-c b / M$. This choice of NS fluxes is such that RR tadpoles due to the fluxes vanish altogether. Equivalently, the 10d Bianchi identity for $F_{2}$ is satisfied without having to add smeared sources $[18,22-24]$. The upshot is that the independent fluxes $c, M, a$ and $b$ are not constrained.

Solving $D_{I} W=0$ the axions are found to be

$$
\operatorname{Im} T=(\lambda+1) \frac{c}{M}, \quad a \operatorname{Im} S+b \operatorname{Im} U=\left(8 \lambda^{2}+1\right) \frac{c}{M},
$$

where $\lambda=(80)^{-1 / 3}$. The saxions are fixed as

$$
s=-\frac{2 c}{a} \lambda t, \quad u=-\frac{6 c}{b} \lambda t, \quad t^{2}=\frac{15 c^{2} \lambda^{2}}{M^{2}} .
$$

Necessarily $c a<0$ and $c b<0$. By taking large $c$ it is possible to attain large vevs for all saxions, as well as small couplings $e^{\phi_{4}}$ and $e^{\phi}$.

The cosmological constant is now given by

$$
\frac{V_{0}}{M_{\mathrm{P}}^{4}}=-\frac{a b^{3}}{120 \mathcal{C} \lambda^{2} c^{2} t^{3}}=-\frac{a b^{3} M^{3}}{120 \sqrt{15} \mathcal{C} \lambda^{5} c^{5}} .
$$

The masses of the canonically normalized moduli have the expected relation to the cosmological constant. We find

$$
M_{\text {mod }}^{2}=\left\{\frac{1}{9}(47+\sqrt{159}), \frac{1}{9}(47-\sqrt{159}), \frac{1}{3}(4+\sqrt{6}), \frac{1}{3}(4-\sqrt{6}),-\frac{2}{3}, 0\right\} \frac{\left|V_{0}\right|}{M_{\mathrm{P}}^{2}} .
$$

The negative eigenvalue is above the Breitenlohner-Freedman bound and the corresponding eigenstate is a combination of $s$ and $u$. The zero eigenvalue is due to the unstabilized combination of axions. Other eigenvectors mix saxions and axions.

The KK mass is determined inserting the vevs of the saxions in (2.8). We obtain

$$
\frac{M_{\mathrm{KK}}}{M_{\mathrm{P}}}=\frac{\left(a b^{3}\right)^{1 / 4}}{\mathcal{C}^{2 / 3} \lambda c t^{3 / 2}}=\frac{\left(a b^{3}\right)^{1 / 4} M^{3 / 2}}{\lambda^{5 / 2} \mathcal{C}^{2 / 3} c^{5 / 2}} .
$$

The ratio to the cosmological constant is then

$$
\frac{M_{\mathrm{P}} M_{\mathrm{KK}}}{\left|V_{0}\right|^{1 / 2}}=\frac{1}{\left(a b^{3}\right)^{1 / 4} \mathcal{C}^{1 / 6}} .
$$

As in the previous case, without incorporating the $10 \mathrm{~d}$ dependence of $\mathcal{C}$ on the geometric fluxes would yield a flux-dependent ratio. Moreover, in this model there is a particularly interesting limit that would seem to violate the strong ADC as we now discuss.

To define the limit correctly, we introduce the parameter $\tau$ which will be sent to infinity. We take

$$
a \sim \tau \quad b \sim \tau, \quad c \sim \tau^{\alpha},
$$


where $\alpha$ is a positive constant to be fixed momentarily. Note that the geometric flux dependent constant is now parametrically different from the naive $4 \mathrm{~d}$ expectation since it goes as $\mathcal{C} \sim \tau^{-6}$. This limit is particularly interesting because it implies

$$
V_{0} \sim-\frac{\tau^{4}}{\tau^{5 \alpha \mathcal{C}}}, \quad \frac{M_{\mathrm{P}} M_{\mathrm{KK}}}{\left|V_{0}\right|^{1 / 2}} \sim \frac{1}{\tau \mathcal{C}^{1 / 6}} .
$$

Clearly $V_{0} \rightarrow 0$ in the $\tau \rightarrow \infty$ limit, both in the naive $4 \mathrm{~d}$ picture and in the full $10 \mathrm{~d}$ solution, provided $\alpha>2$. It can be checked that the limit leads to large volume and small couplings $e^{\phi_{4}}$ and $e^{\phi}$. Now, from the purely $4 \mathrm{~d}$ EFT point of view one could erroneously claim that (2.24) embodies a counterexample to the strong ADC, since in the $V_{0} \rightarrow 0$ limit we observe a parametric deviation from $M_{\mathrm{P}} M_{\mathrm{KK}} \sim\left|V_{0}\right|^{1 / 2}$. Once again, this seems to be an artifact of the $4 \mathrm{~d}$ calculation of the KK scale. Taking into account the full 10d geometry through the proper value of $\mathcal{C}$, the dependence on the geometric fluxes of the ratio $(2.22)$ identically drops out and there is no parametric separation between the two scales. Finally, note that even though the limit $\tau \rightarrow \infty$ seems dangerous from the purely $4 \mathrm{~d}$ EFT, it does not exhibit what is usually referred to as scale separation since ignoring $\mathcal{C}$ the $\mathrm{KK}$ scale becomes arbitrarily lower than $\left|V_{0}\right|^{1 / 2}$ (instead of higher), thereby bringing the EFT out of control. Still, this shows a limit in which the full 10d solution crucially modifies the naive expectations from the lower dimensional EFT in a way consistent with the strong ADC. Moreover, once again, the relation between the corrected KK scale and $\left|V_{0}\right|^{1 / 2}$ happens to be captured by the mass of the moduli, since even in the naive $4 \mathrm{~d}$ picture we have $M_{\mathrm{P}} M_{\text {mod }}=\mathfrak{n}\left|V_{0}\right|^{1 / 2}$.

\section{A class of type IIB vacua}

In this section we look into a class of supersymmetric type IIB vacua which are dual to the ones studied in the previous section. As we will see, from the naive approximation of the KK scale available in the $4 \mathrm{~d}$ EFT, these vacua seem to exhibit both scale separation and a behavior that contradicts the strong ADC. In these cases, since they include non-geometric fluxes we do not have a full 10d solution which we can use to compute the corrected KK scale and check whether it is modified in such a way that scale separation is not achieved and the strong ADC fulfilled. Our strategy for these vacua is then to use their type IIA duals, whose 10d solution geometry we explained in the previous section, and estimate the corrected KK scale by careful identification on the IIA side.

The non-geometric IIB theory is obtained by performing three T-dualities along the three $x$ directions of the twisted torus in IIA [29]. In the $4 \mathrm{~d} \mathcal{N}=1$ theory, we again restrict ourselves to the case $T_{i}=T$ and $U_{i}=U$. The scalar potential is calculated by means of the standard eq. (2.2). The Kähler potential takes the form

$$
K=-\log (S+\bar{S})-3 \log (U+\bar{U})-3 \log (T+\bar{T}) .
$$

The superpotential is derived by replacing $T \leftrightarrow U, S \rightarrow S$ in (2.6). Thus

$$
W=e_{0}+3 i e U+3 c U^{2}+i M U^{3}+i h_{0} S-3 i h T-3 a S U-3 b T U .
$$


The coefficients are labeled as before but now $h$ and $b$ have a different interpretation in terms of non-geometric fluxes in type IIB. We have not included a possible flux-dependent normalization constant, but will keep in mind that it might be present.

For completeness we recall the relation between the Planck and the string masses in IIB compactifications, namely

$$
M_{\mathrm{s}}^{2}=\frac{g_{s}^{1 / 2} M_{\mathrm{P}}^{2}}{2 \pi \mathcal{V}}
$$

where $g_{s}=e^{\phi}=s^{-1}$ and $\mathcal{V}=t^{3 / 2}$. At the level of the EFT, the KK scale is approximated by

$$
M_{\mathrm{KK}} \sim \frac{M_{\mathrm{s}}}{V_{6}^{1 / 6}} \sim \frac{M_{\mathrm{P}}}{\mathcal{V}^{2 / 3}},
$$

where we used that in IIB, $V_{6}=e^{3 \phi / 2} \mathcal{V}$. In the above we have neglected warp factors. Let us remark that at this point all these relations refer to the compact manifold consisting of a flat toroidal orientifold with (non-geometric) fluxes, which is the 4d EFT framework. In this case with non-geometric fluxes the 10d picture is not even in terms of standard spacetime.

\subsection{Example 3, $M=0$}

To simplify the discussion, we again turn off the fluxes $h_{0}, h$ and $e$, and recall that the flux $e_{0}$ is not constrained by any tadpole. As expected by consistency with the IIA dual, there exists a supersymmetric vacuum, see also [30]. The axions are fixed at values

$$
\operatorname{Im} U=0, \quad a \operatorname{Im} S+b \operatorname{Im} T=0,
$$

whereas the saxions are stabilized according to

$$
s=\frac{2 c}{a} u, \quad t=\frac{6 c}{b} u, \quad u^{2}=\frac{e_{0}}{9 c} .
$$

The value of the potential at the minimum is given by

$$
\frac{V_{0}}{M_{P}^{4}}=-\frac{a b^{3}}{128 c^{2} u^{3}}=-\frac{27 a b^{3}}{128 c^{1 / 2} e_{0}^{3 / 2}},
$$

which coincides with eq. (2.13) after changing $t \rightarrow u$ and setting $\mathcal{C}=1$. The masses of the moduli are given by (2.14). Using (3.4), the EFT approximation of the KK scale yields

$$
\frac{M_{\mathrm{KK}}}{M_{\mathrm{P}}} \sim \frac{1}{t} \sim \frac{1}{\left(2 c e_{0}\right)^{1 / 2}} .
$$

The ratio of $M_{\mathrm{KK}}$ to the cosmological constant scale takes the form

$$
\frac{M_{\mathrm{P}} M_{\mathrm{KK}}}{\left|V_{0}\right|^{1 / 2}} \sim \frac{e_{0}^{1 / 4}}{c^{1 / 4}(a b)^{1 / 2}},
$$

which is in agreement with [30]. 
In this example, taking $e_{0}$ to be large implies a large complex structure point in which the string coupling is small, so the EFT stays within its regime of validity. Moreover, upon taking the limit $e_{0} \rightarrow \infty$, scale separation would hold since the KK mass becomes arbitrarily larger than the cosmological constant scale. In addition, since in this limit $V_{0} \rightarrow 0$, the strong ADC would also be violated. We claim that this is again an artifact of the wrong approximation for $M_{\mathrm{KK}}$. The supporting arguments rely on the T-duality with the type IIA model of section 2.1, which has a clear 10d interpretation as explained before.

As already mentioned, to go from IIA to IIB, one performs three T-dualities along the three $x$ directions of the twisted torus. Upon this transformation, the sizes associated to the three $y$ directions remain untouched, so that the KK scale asociated to the $y$ directions in the IIA side maps to the KK scale along the same directions in the IIB dual. Knowing the correct KK scale asociated to the $y$ direction in the IIB side, we can in fact justify that it will be the dominant one. The reason is that the large complex structure limit corresponds (for rectangular tori) precisely to having typical length along $y$, much larger than the one along $x$, say $R_{y} \gg R_{x}$. Hence, the lowest KK scale in the IIB side will be given by just dualizing the IIA KK scale associated to $R_{y}$. In both IIA and IIB we can define $M_{\mathrm{KK}}^{y} \sim M_{\mathrm{s}} / R_{y}$. The dependence on the moduli in the two theories is different but straightforward to derive. ${ }^{2}$

In IIB we just find

$$
\frac{M_{\mathrm{KK}}^{y}}{M_{\mathrm{P}}} \sim \frac{1}{t u^{1 / 2}} \sim \frac{b}{c^{1 / 4} e_{0}^{3 / 4}} .
$$

This yields the ratio

$$
\frac{M_{\mathrm{P}} M_{\mathrm{KK}}^{y}}{\left|V_{0}\right|^{1 / 2}} \sim \frac{1}{(a b)^{1 / 2}},
$$

in which the dependence on $e_{0}$ and $c$ has canceled so that the parametric scale separation, as well as the parametric violation of the strong ADC disappear. There is still the dependence on the fluxes $a, b$. Now, in IIA we can just read off the result for $M_{\mathrm{KK}}^{y} / M_{\mathrm{P}}$ from (2.15) by setting $a=b$. This gives a result that coincides with (3.10) except for a factor of $\mathcal{C}^{2 / 3}$ in the denominator. Invoking T-duality we can say that this factor is missing in IIB and should be included. Applying the same reasoning to $V_{0}$, the flux dependence in the ratio will cancel. This was not done before because we were only using the 4d EFT, which did not predict the factors of $\mathcal{C}$. Note however that including these factors would not alter the previous discusion involving the $e_{0} \rightarrow \infty$ limit, since $\mathcal{C}$ is independent of $e_{0}$.

In the end we can distinguish three levels of refinement. In the first one, when the KK scale is approximated by (3.8), we seem to violate both the ASSC and the strong ADC parametrically. After realizing that the isotropic approximation is not justified and using the dominant length scale, $R_{y}$, to calculate the KK mass as in (3.10), we observe that the parametric violation of both conjectures disappears, but still there is some dependence in the fluxes $a$ and $b$. Finally, if we take into account the full $10 \mathrm{~d}$ geometry of the dual and dualize the KK scale along the directions that remain untouched, as well as $V_{0}$, we find a flux independent relation $M_{\mathrm{P}} M_{\mathrm{KK}} \sim\left|V_{0}\right|^{1 / 2}$.

\footnotetext{
${ }^{2}$ See e.g. [31] for a full correspondence between the KK and winding scales in toroidal compactifications of type IIA and type IIB.
} 


\section{Final comments}

The purpose of this paper has been to test the ASSC and ADC within the context of $4 \mathrm{~d}$ toroidal orbifold orientifolds in type II string theory. Concerning the ASSC, it was already known from many examples that, at the level of known 10d compactifications, such a separation does not exist. Here we have focused however on the effective $4 \mathrm{~d}$ effective action description of $\mathrm{AdS}_{4}$ type II vacua, in which some examples seem to violate the conjecture. We have seen that models where the ASSC conjecture seems to be violated at the $4 \mathrm{~d}$ effective theory level, do not contradict it once one takes into account the backreaction within a complete 10d formulation. The same happens with the related ADC, which claims a behavior $M_{\mathrm{KK}} \propto\left|V_{0}\right|^{\gamma}$ as $V_{0} \rightarrow 0$, with $\gamma$ positive and equal to $1 / 2$ in the supersymmetric case. Using the effective field theory approach, a violation is observed in a number of examples. However, after considering the backreaction of the geometry when a 10d uplift exists, no violation appears.

One interesting property that we observe, at least in this class of models, is that we actually find $M_{\mathrm{KK}}^{2}=\mathfrak{c}\left|V_{0}\right| / M_{\mathrm{P}}^{2}$ with $\mathfrak{c}$ a flux-independent constant. This goes beyond the strong $\mathrm{ADC}$ in the sense that there is no need to set the non-leading fluxes of the compactification to small values, it is valid for any value of the fluxes. This behavior is universal in this class of models and the only model dependence is in the numerical constant c. In this sense the behavior is like the one of the Breitenlohner-Freedman bound, which is also universal [27]. Another interesting property, already noticed in [10], is that with respect to the cosmological constant, the moduli masses behave exactly like $M_{\mathrm{KK}}$, up to numerical factors. Thus, in AdS vacua, the ratio $M_{\mathrm{mod}}^{2} /|\Lambda|$ determined from the effective potential gives us automatically the ratio $M_{\mathrm{KK}}^{2} /|\Lambda|$ in the theory, without the need of an explicit computation. It would be interesting to understand these points further.

Although we focused on $\mathcal{N}=1$ supersymmetric models with a $10 \mathrm{~d}$ lift, we also considered non-supersymmetric vacua in the $4 \mathrm{~d}$ EFT. In this latter case we found that the mass scales also follow the pattern $M_{\mathrm{KK}} \sim M_{\text {mod }} \sim\left|V_{0}\right|^{1 / 2}$, so that there is no scale separation and the strong ADC is satisfied.

There are models like the specific $M \neq 0$ examples with no geometric fluxes in $[12,13]$ which seem to violate both conjectures if analyzed from the $4 \mathrm{~d}$ point of view. These examples admit a 10d extension with smeared sources, but a solution with localised O6planes is not guaranteed [14]. In any case, the evidence found for the examples considered here seem to suggest that in that case also the mass of the moduli captures the effective KK scale, and that again the ASSC and strong ADC hold.

\section{Acknowledgments}

We would like to thank R. Blumenhagen, M. Brinkmann, F. Marchesano, M. Montero, E. Palti, A. Uranga, T. Van Riet and I. Valenzuela for useful discussions. This work is supported by the Spanish Research Agency (Agencia Estatal de Investigacion) through the grant IFT Centro de Excelencia Severo Ochoa SEV-2016-0597, and by the grants FPA201565480-P and PGC2018-095976-B-C21 from MCIU/AEI/FEDER, UE. A.H. is supported 
by the Spanish FPU Grant No. FPU15/05012 and would like to thank the Max Planck Institute for Physics in Munich for their hospitality during completion of this work. A.F. thanks the IFT UAM-CSIC for hospitality and support during completion of this work.

Open Access. This article is distributed under the terms of the Creative Commons Attribution License (CC-BY 4.0), which permits any use, distribution and reproduction in any medium, provided the original author(s) and source are credited.

\section{References}

[1] C. Vafa, The String landscape and the swampland, hep-th/0509212 [INSPIRE].

[2] T.D. Brennan, F. Carta and C. Vafa, The String Landscape, the Swampland and the Missing Corner, PoS (TASI2017) 015 (2017) [arXiv: 1711.00864] [INSPIRE].

[3] E. Palti, The Swampland: Introduction and Review, Fortsch. Phys. 67 (2019) 1900037 [arXiv: 1903.06239] [INSPIRE].

[4] F.F. Gautason, M. Schillo, T. Van Riet and M. Williams, Remarks on scale separation in flux vacua, JHEP 03 (2016) 061 [arXiv:1512.00457] [INSPIRE].

[5] D. Lüst, E. Palti and C. Vafa, AdS and the Swampland, Phys. Lett. B 797 (2019) 134867 [arXiv: 1906.05225] [INSPIRE].

[6] M.J. Duff, B.E.W. Nilsson and C.N. Pope, Kaluza-Klein Supergravity, Phys. Rept. 130 (1986) 1 [INSPIRE].

[7] M.R. Douglas and S. Kachru, Flux compactification, Rev. Mod. Phys. 79 (2007) 733 [hep-th/0610102] [INSPIRE].

[8] D. Tsimpis, Supersymmetric AdS vacua and separation of scales, JHEP 08 (2012) 142 [arXiv: 1206.5900] [INSPIRE].

[9] F.F. Gautason, V. Van Hemelryck and T. Van Riet, The Tension between $10 D$ Supergravity and dS Uplifts, Fortsch. Phys. 67 (2019) 1800091 [arXiv: 1810.08518] [InSPIRE].

[10] R. Blumenhagen, M. Brinkmann and A. Makridou, Quantum Log-Corrections to Swampland Conjectures, JHEP 02 (2020) 064 [arXiv:1910.10185] [INSPIRE].

[11] G. Villadoro and F. Zwirner, $N=1$ effective potential from dual type-IIA D6/O6 orientifolds with general fluxes, JHEP 06 (2005) 047 [hep-th/0503169] [INSPIRE].

[12] O. DeWolfe, A. Giryavets, S. Kachru and W. Taylor, Type IIA moduli stabilization, JHEP 07 (2005) 066 [hep-th/0505160] [INSPIRE].

[13] P.G. Camara, A. Font and L.E. Ibáñez, Fluxes, moduli fixing and MSSM-like vacua in a simple IIA orientifold, JHEP 09 (2005) 013 [hep-th/0506066] [INSPIRE].

[14] B.S. Acharya, F. Benini and R. Valandro, Fixing moduli in exact type IIA flux vacua, JHEP 02 (2007) 018 [hep-th/0607223] [INSPIRE].

[15] E. Palti, G. Tasinato and J. Ward, WEAKLY-coupled IIA Flux Compactifications, JHEP 06 (2008) 084 [arXiv:0804.1248] [INSPIRE].

[16] F. Marchesano and J. Quirant, A Landscape of AdS Flux Vacua, JHEP 12 (2019) 110 [arXiv: 1908.11386] [INSPIRE]. 
[17] T.W. Grimm, C. Li and I. Valenzuela, Asymptotic Flux Compactifications and the Swampland, arXiv:1910.09549 [INSPIRE].

[18] G. Aldazabal and A. Font, A Second look at $N=1$ supersymmetric AdS $S_{4}$ vacua of type IIA supergravity, JHEP 02 (2008) 086 [arXiv:0712.1021] [INSPIRE].

[19] B.S. Acharya, F. Denef, C. Hofman and N. Lambert, Freund-Rubin revisited, hep-th/0308046 [INSPIRE].

[20] P. Koerber and L. Martucci, D-branes on AdS flux compactifications, JHEP 01 (2008) 047 [arXiv: 0710.5530] [INSPIRE].

[21] C. Caviezel, P. Koerber, S. Körs, D. Lüst, D. Tsimpis and M. Zagermann, The Effective theory of type IIA AdS $S_{4}$ compactifications on nilmanifolds and cosets, Class. Quant. Grav. 26 (2009) 025014 [arXiv:0806.3458] [INSPIRE].

[22] D. Lüst and D. Tsimpis, Supersymmetric AdS $S_{4}$ compactifications of IIA supergravity, JHEP 02 (2005) 027 [hep-th/0412250] [INSPIRE].

[23] K. Behrndt and M. Cvetič, General $N=1$ supersymmetric flux vacua of (massive) type IIA string theory, Phys. Rev. Lett. 95 (2005) 021601 [hep-th/0403049] [INSPIRE].

[24] K. Behrndt and M. Cvetič, General $N=1$ supersymmetric fluxes in massive type IIA string theory, Nucl. Phys. B 708 (2005) 45 [hep-th/0407263] [INSPIRE].

[25] M. Graña, R. Minasian, M. Petrini and A. Tomasiello, A Scan for new $N=1$ vacua on twisted tori, JHEP 05 (2007) 031 [hep-th/0609124] [INSPIRE].

[26] L.E. Ibáñez and A.M. Uranga, String theory and particle physics: An introduction to string phenomenology, Cambridge University Press (2012) [INSPIRE].

[27] P. Breitenlohner and D.Z. Freedman, Positive Energy in anti-de Sitter Backgrounds and Gauged Extended Supergravity, Phys. Lett. 115B (1982) 197 [InSPIRE].

[28] D. Lüst, F. Marchesano, L. Martucci and D. Tsimpis, Generalized non-supersymmetric flux vacua, JHEP 11 (2008) 021 [arXiv:0807.4540] [INSPIRE].

[29] G. Aldazabal, P.G. Camara, A. Font and L.E. Ibáñez, More dual fluxes and moduli fixing, JHEP 05 (2006) 070 [hep-th/0602089] [INSPIRE].

[30] R. Blumenhagen et al., A Flux-Scaling Scenario for High-Scale Moduli Stabilization in String Theory, Nucl. Phys. B 897 (2015) 500 [arXiv:1503.07634] [INSPIRE].

[31] A. Font, A. Herráez and L.E. Ibáñez, The Swampland Distance Conjecture and Towers of Tensionless Branes, JHEP 08 (2019) 044 [arXiv: 1904.05379] [INSPIRE]. 